This item was submitted to Loughborough's Research Repository by the author.

Items in Figshare are protected by copyright, with all rights reserved, unless otherwise indicated.

\title{
An interoperable semantic service toolset with domain ontology for automated decision support in the end-of-life domain
}

\section{PLEASE CITE THE PUBLISHED VERSION}

https://doi.org/10.1016/j.future.2020.06.008

\section{PUBLISHER}

Elsevier

\section{VERSION}

AM (Accepted Manuscript)

\section{PUBLISHER STATEMENT}

This paper was accepted for publication in the journal Future Generation Computer Systems and the definitive published version is available at https://doi.org/10.1016/j.future.2020.06.008.

\section{LICENCE}

CC BY-NC-ND 4.0

\section{REPOSITORY RECORD}

Pease, Sarogini, Richard Sharpe, Kate Van-Lopik, Eleni Tsalapati, Paul Goodall, Robert I.M. Young, Paul Conway, and Andrew West. 2020. "An Interoperable Semantic Service Toolset with Domain Ontology for Automated Decision Support in the End-of-life Domain”. Loughborough University. https://hdl.handle.net/2134/13611143.v1. 


\title{
An Interoperable Semantic Service Toolset with Domain Ontology for Automated Decision Support in the End-of-Life Domain
}

\author{
Sarogini Grace Pease, Richard Sharpe, Kate van Lopik, Eleni Tsalapati, Paul Goodall, Bob Young, Paul Conway \\ and Andrew West \\ Wolfson School of Mechanical, Electrical and Manufacturing Engineering, Loughborough University, Loughborough, Leicestershire, LE11 3TU
}

$U K$

\begin{abstract}
In product-diverse, end-of-life (EoL) production lines the relevant markets, competitors and customer bases continuously change as new products are processed. The resale market itself changes with the influx of new products, as well as hardware and software discontinuations. Competitive business decision making is often performed by a human operator and may not be timely or fully informed. These are decisions such as whether to perform a high cost repair or recycle a product or whether to use a batch of parts in repair or sell them on can be used to optimise product life-cycle management (PLM) and profit margins. A real-time decision making capability can reduce the risk of performing non-profitable processing. The novel contribution of this work is an interoperable semantic decision support toolset that is necessary to enable a capability for timely EoL decisions based on complete knowledge on profitability, predicted pricing and cost-of-production. Many decision support systems have been proposed for the EoL domain, but a lack of interoperability and use of unstructured knowledge bases has led to decisions based on knowledge that is not up to date. Using formalised, semantic technologies offers sustainable decision making in this volatile and increasingly competitive domain.
\end{abstract}

Keywords:

\section{Introduction}

The key competitive business decision in EoL product recovery is whether to process (redeploy, refurbish or remanufacture) or recycle a product. The decision hinges on total processing cost and appropriate pricing of the product. In contrast to the Beginning-of-Life (BoL) manufacturing domain, the EoL profitability decision has to be repeatedly re-evaluated during the route from product inspection to recovery. It is a complex decision as products in EoL lines will be of diverse types, usage, qualities and market desirability. Decisions are time-critical as the applicable retail market, competitors and customer base will change dynamically as the line runs. Operator decisions on whether to recycle or conduct potentially high-cost refurbishment, or on whether to use components for repairs or sell them on, can be more timely, informed and appropriately made when supported by an automated, intelligent system. Underpinning this with an interoperable, semantic knowledge base can future-proof the system to gather complete, upto-date knowledge in deciding appropriate pricing as the

Preprint submitted to Future Generation Computer Systems market changes [1]. PLM activities can then be optimised to reduce the performance of non-profitable processing when this costs more than the product price.

An EoL artificial intelligence (AI) decision support toolset is proposed in this paper. This combines two AI branches; semantic and machine learning technologies with the goal of accurate, timely prediction of product profitability. The main research contributions of this paper are:

1. Design of an interoperable semantic service toolset with a novel approach to distributed decision support that is reusable across the EoL domain.

2. Automation of knowledge update using a semantically enriched formal schema, or Web Ontology Language (OWL) 2 / RDF (Resource Description Framework) ontology, describing the EoL domain, mapped to the BFO (Basic Formal Ontology) upper-level ontology to offer re-usability outside the applied sub-domain of a Waste Electrical and Electronic Equipment (WEEE) recovery or- 
ganisation.

3. Validation of the toolset for remote deployment and querying of the linked-data and initial demonstration of the decision-making capabilities.

\section{Motivation}

A profitability decision will be made at several points during EoL processing: this decides the costeffectiveness of refurbishment or repair over recycling. The decision incorporates determining the EoL processing costs and an appropriate product price and any business and regulatory constraints that will control whether recovery of profit is possible (e.g. controls on medical goods or that sellers check that a phone International Mobile Equipment Identity (IMEI) is not reported as stolen). Pricing strategies applied may focus on profitability, increasing market share or defence against competition. Competitor prices proliferate gradually into the public domain following a product release and can vary across a product family as features are introduced and retired. Figure 1 proposes the knowledge that is determinant in the profitability decision $[2,3]$. There is a need for interoperability with a wide range of resources to collate accurate information on these factors and to maintain this accuracy over time.

Figure 2 outlines decisions performed within a partner WEEE EoL recovery facility: blue diamonds are the decisions performed by the EoL worker. Products are first logged for tracking and if not declared during visual inspection as beyond repair and recycled, a profitability decision is made. The worker may access knowledge bases ${ }^{1}$ to establish if a product has been stolen or a phone is locked to a network or to check recycle value. Visual inspection identifies any obvious external damage and any repairs required. The product must pass safety tests, e.g. Portable Appliance Test (PAT), a data erasure process and a functional test; that the device functions as designed to. If repairs fail the product is recycled, otherwise it returns to visual inspection. Where the organisation does not have the expertise or components for the repair (unrepairable) the product may be stripped and recycled or sold in an unrepaired condition.

Knowledge that is critical to the profitability decision is gathered in a heterogeneous range of knowledge bases that a worker must manually access. Some may be held internally by their organisation, including wikis or Intranet sites, some will be publicly available via websites and API's. Knowledge can be structured, such as

\footnotetext{
1 e.g. https://www.checkmend.com/uk/
}

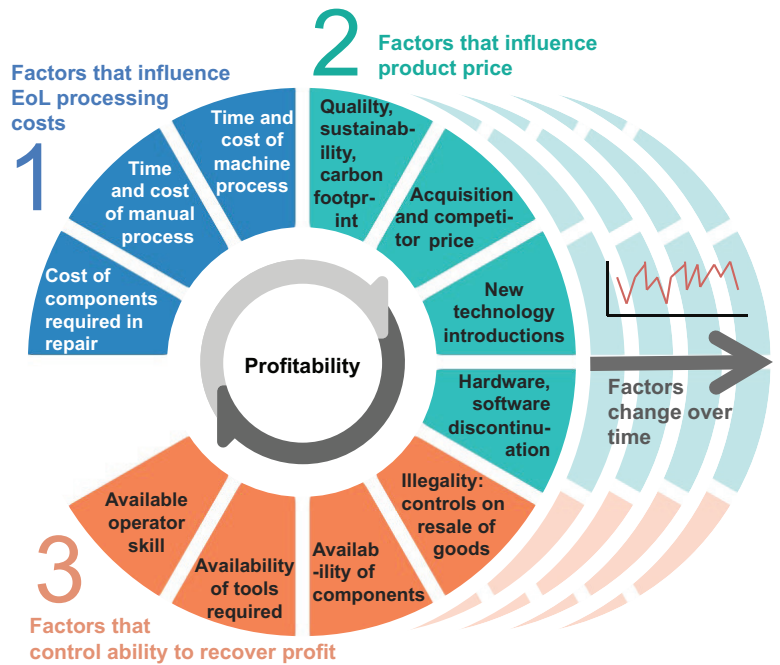

Figure 1: Interoperability with Sources of Knowledge to Make Appropriate EoL Profitability Decisions

databases or CSV files or unstructured data lakes of, for example, documents and images. Pricing propriety is at risk of varying with the performance, training and experience of the operator as well as with outdated knowledge and human error introduced during manual searching.

Timely decision support automation could minimise variability in pricing propriety $[4,5,6]$. However, the operator will still make the final choice on whether to follow a direction provided by automated decision support. Such human-automation trust will depend on the system being consistently proficient in handling high risk decisions. This can be aided by indicators on system confidence in the source of knowledge. Attack resistant trust metrics are commonly used to measure trust in other nodes or users and quantify prediction confidence. These include Advogato, the Google PageRank algorithm or EigenTrust used in peer-to-peer (P2P) networks. Links and interactions between trusted objects then support increasing trust in other objects [7, 8]. There is a strong interdependence between knowledge management and decision support. Knowledge is acquired and deployed to form the basis of a decision [9]. Interoperability with EoL knowledge bases available can provide robustness to change; to an increasing degree if these knowledge bases are managed and up-todate [10].

The variety of terms used in existing EoL knowledge bases as well as the heterogeneous access and data models are a restriction to interoperability that can be mitigated using formal logic and semantic technologies. 


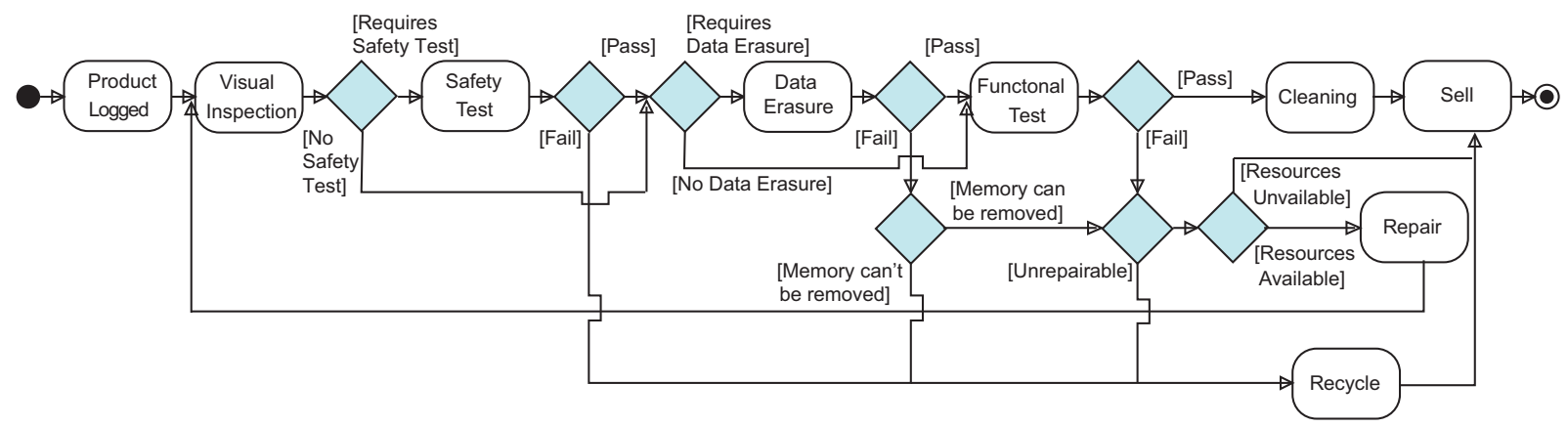

Figure 2: Activity Diagram: EoL WEEE Recovery Organisation

Formal ontologies are composed of entities interlinked with typed relationships e.g. using RDF and and semantics are defined using a knowledge representation language e.g. OWL 2. The use of semantic web technologies ${ }^{2}$ further supports remote and automated query answering of valuable implicit knowledge from the information that exists in the knowledge base as well as checking for consistency and completeness of the knowledge base [5].

Ontologies have been used to enhance traditional decision support tools by providing a shared and common understanding of domain knowledge within a structured and formal representation [11]. This semantic interoperability enables continued and future updates to a knowledge base that can operate in conjunction with heterogeneous sources of knowledge. Ensuring that knowledge sharing is flexible, scalable and interoperable depends on a conceptual representation that is semantically consistent at every level $[12,13,11,14,15]$.

\section{Background}

Decision support for EoL is an open research area, with many solutions designed to evaluate profitability in the processes of remanufacture, recondition, repair, recycle and disposal of products. The first three processes conduct restorative acts to ensure the product is functional and meets quality standards. Recycling recovers the material value of a product, while disposal incurs a cost. Few of these proposed approaches to profitability or pricing decisions have benefited from interoperable semantic knowledge management or used semantics to provide access to services.

\footnotetext{
${ }^{2}$ https://www.w3.org/standards/semanticweb/
}

\subsection{EoL Profitability Decisions}

The authors in [16] developed a non-linear decision support model to predict processing costs, based on component condition and PLM quality information, to decide whether to process or dispose of turbocharger components. In [17] a tool using genetic and ant-colony search algorithms was used to support the decision to store, cannibalise or repair CPU and RAM components. Stochastic simulation has been used to weigh up processing costs for EoL electronic waste against recovery value for individual components, when there are some fixed and some variable costs associated with each disassembly option [18]. However, the cost of disposal and associated processes, such as wiping hard drives is not considered. Web-based recycling decision support and a PLM have been used to connect an operator with a relational database of disassembly time and cost, potential recycling profit and environmental impact assessment [19]. These approaches have incorporated current pricing into the tools and offer no common representation of the domain knowledge relating to processing profitability. This limits the capability to provide consistent pricing validity over time or tuned to changes in the market.

The authors in [1] developed a semantic framework to promote sharing traceability, pricing, material, legal and technical data during WEEE EoL processing. Remote queries and methods of service orchestration and matchmaking mean that the system could be extended to decision support. A unique global ontology is created to act as an upper level ontology and an application ontology can be manually updated according to it. This system would have benefited from being developed in line with a more widely used upper ontology [20], providing increased semantic interoperability. Upper-level ontologies, also known as top-level and foundation ontologies, consist of key concepts that are common across differ- 
ent domains. Domain ontologies describe a specific domain and refer to identifiable entities. Without a full semantic representation of the WEE EoL domain there is no shared understanding of meaning and thus limited scalability to other EoL domains. An ontology was also developed by [12] for use in a closed-loop PLM in the automotive industry. Original UML diagrams were used to encapsulate the knowledge on the physical product, required equipment and personnel resources. The system used a reasoner to manage inconsistency and could be extended to include decision making functionality.

The research outlined in this paper aims to ensure interoperability through a multilayer approach: an EoL domain ontology (applied to the case study of WEEE recovery) that conforms to a widely used upper level ontology, BFO [21, 20, 22]. BFO has been successfully used for ontology development, as a basis of the Common Core Ontologies ${ }^{3}$ and to divide up the biomedical domain into interoperable sub-domain ontologies. The Industry 4.0 and semantic web paradigm shifts have led to a proliferation of orphan ontologies that lack consistency between definitions and rules describing each domain. However, mapping between legacy and contemporary domain ontologies is supported when they are derived from the top-down, using one upper ontology [23].

\subsection{Interoperable Services}

Combining the representation and reasoning capabilities of semantic web technologies with service-oriented control can offer semantic interoperability to the software components in a toolset. Semantic service discovery where application components expose service access, makes it possible for agents to autonomously engage with common interfaces [24]. The toolset, proposed in Section 4, uses this approach to manage interaction with heterogeneous sources and applications.

A range of multi-layer semantic architectures have used networking layers, middleware and interfaceaccess layers for interconnectivity and discovery of services $[25,24]$. The authors in [26] employed semantic process mediation to ensure choreographed interoperability between services. Service buses have been applied to generalise interface access and perform discovery, routing, mapping and parsing. This supports the advertisement of functionality rather than just interfaces, such as a Web Services Description Language (WSDL) descriptor [27, 28, 29, 28]. Ontology representation of functionality is key to accessing this knowl-

\footnotetext{
${ }^{3}$ https://github.com/CommonCoreOntology
}

edge, through definition of inputs, outputs, invocation processes and mapping services [27].

An interoperability framework was proposed in [30], converting information output by unmanned aircraft system models from JavaScript Object Notation (JSON) format into a bespoke ontology and then querying this with SPARQL to produce an MS Excel design and tradeoff decision table. The ontology produced was aligned with BFO but only encapsulated concepts relating to a specific system. Thus the framework offered interoperability with a range of sources but not scalability to multiple systems or a whole domain.

Semantic architectures still have performance challenges in terms of scaling data output, multi-level heterogeneity, conflicts between applications and correction of human errors in metadata $[31,32,1]$. The authors in [33] proposed an ontology framework for intelligent data analysis to manage validation, representation and interpretation of data from heterogeneous sources. The observed data were sanitised for noise, outliers and input errors and then stored. The metadata was then attached; the set included recorded precision and range of the devices used. Correspondingly, the toolset proposed in this paper aims to limit the need for large data output as well as human input. Some reconciliation of structural conflicts and data sanitisation will be conducted by a semantic service bus and domain and data conflicts by the necessity to comply with domain and upper-level ontologies.

\subsection{Interoperable Ontologies}

An ontology is a conceptual schema that formally describes the domain of interest. It consists of atomic classes, individuals and properties. Classes represent abstract groups, sets, or collections of objects. Individuals represent real-world objects, and properties relate objects to each other (object properties) or to data values (data properties). In the WEEE EoL domain, the class Product can be used to group various instances of products, e.g. models of mobile phones or phone screens. Individuals of a process class could be specific processes, process $_{x_{i}}$, process $x_{j}$, where $x_{i}, x_{j}$ represent an identification number, taking place at a timestamp. To express formally that a product participates in some manufacturing process, the object property participatesIn can be defined, with a domain of the class Product and a range of the class process. The datatype property isIdentifiedBy can be used to attribute products and processes with an identification code of integer value, e.g. process $S_{\mid D 18}$.

For more elaborate descriptions of a domain, relationships between terms, or axioms are defined. In this 


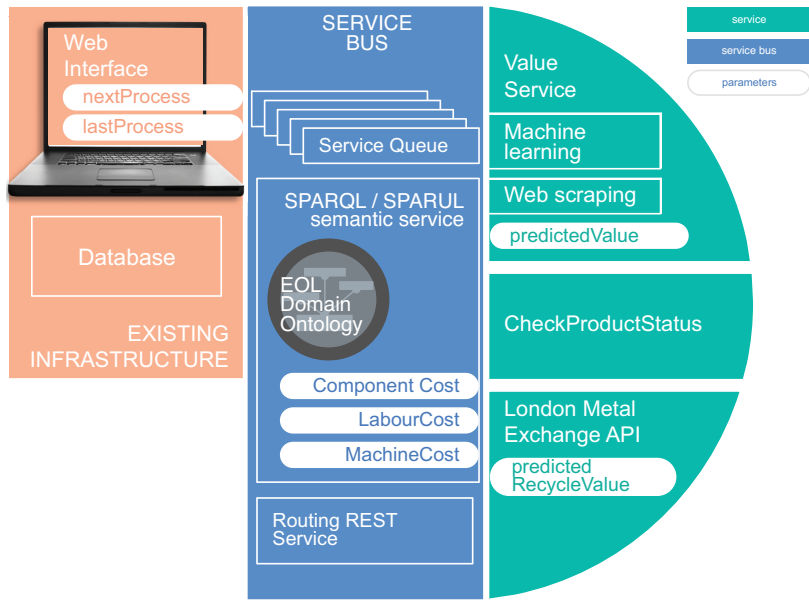

Figure 3: Toolset Services and Inferred Output Parameters

work the user-friendly syntax for expressing axioms is adopted, Manchester syntax, which uses the constructor isSubClassOf. For instance, the axiom ActOfAssembly isSubClassOf process, informally expresses that every individual that belongs to the ActOfAssembly class also belongs to the process class: every assembly is a process. A subclass always inherits the properties of its superclasses. Other constructors, or, and, only, some, value, min, one can be used to define more complex axioms, as in the following example that expresses that every ActOfDisassembly process can be followed by either a recycle or a repair process:

ActOfDisassembly isSubClassOf isFollowedBy only (ActOfRecycling or ActOfRepair)

The authors in [34] used a SPARQL server to interrogate a developed ontology, in deciding whether to reuse or repair construction parts, but didn't gather knowledge from diverse sources or offer extensibility through adherence to an upper ontology. However, using analytical modelling enabled weighting of the output. The output was also augmented by human expert knowledge in weighting characteristics although the number of required sources and level of skill were not defined.

Automated decision making has been proposed using a mechanical disassembly domain ontology combined with case based reasoning to generate a full disassembly process [35]. The use of diverse external sources for population of the domain ontology was suggested, but not implemented. [31] proposed a data integrating gateway between the knowledge base and the shop floor. While the gathering of knowledge was not completely automated, the ontology was updated with KPI's during assembly and reconfiguration planning. Whereas in
[6] combined web crawling to fully automate the update of an ontology, building a series of reusable collection routes from across the web.

A self-contained decision making ontology was proposed in [13] and theoretically applied to the problem of replacing components when a service warranty is triggered but no fault is found with the product. The cost of replacing a component as well as the output of stress testing were considered in deciding whether there was a fault and whether to refurbish or recycle.

\section{Interoperable Semantic Service Toolset}

This paper proposes an innovative toolset design that supports distributed decision support and a formal domain ontology applied to the sub-domain of a WEEE recover. A semantic service architecture that is reusable across the EoL domain can meet industry-specific requirements with services defined according to business process requirements, client needs and toolset interoperability. Previous proposed EoL decision support approaches have offered the capability to present processing costs to the operator on the basis of a fixed knowledge base that is not updated over time. These systems use services and algorithms embedded on devices and limited to unconventional architectures that can be inflexible to business requirements and technological progress.

The proposed toolset functionality, illustrated in Figure 3 , has been designed to be interoperable with new and legacy infrastructure of an EoL organisation by being agnostic to any database schema. Initial integration would require development or modification of only the existing database access web interface of the user to send product information to the ontology in the form of triggering SPARQL (SPARQL Protocol and RDF Query Language) and SPARUL (SPARQL/Update) queries. As a product is processed in a EoL facility, the unique ID and processes completed (e.g. disassembly, replace RAM, cleaning, assembly) would be input as the product progressed through the line.

This connects to a service bus that provides generic infrastructure-service access to client-service functionality through mediation, orchestration, routing and abstraction of information and messages, that can support the modular addition of new services. A routing RESTful (Representational State Transfer) service is responsible for directing parameters to the SPARQL/SPARUL service or on to the specific service connected to the service bus that requires them. The SPARQL/SPARUL service, or endpoint handles updates and queries to the ontology. 


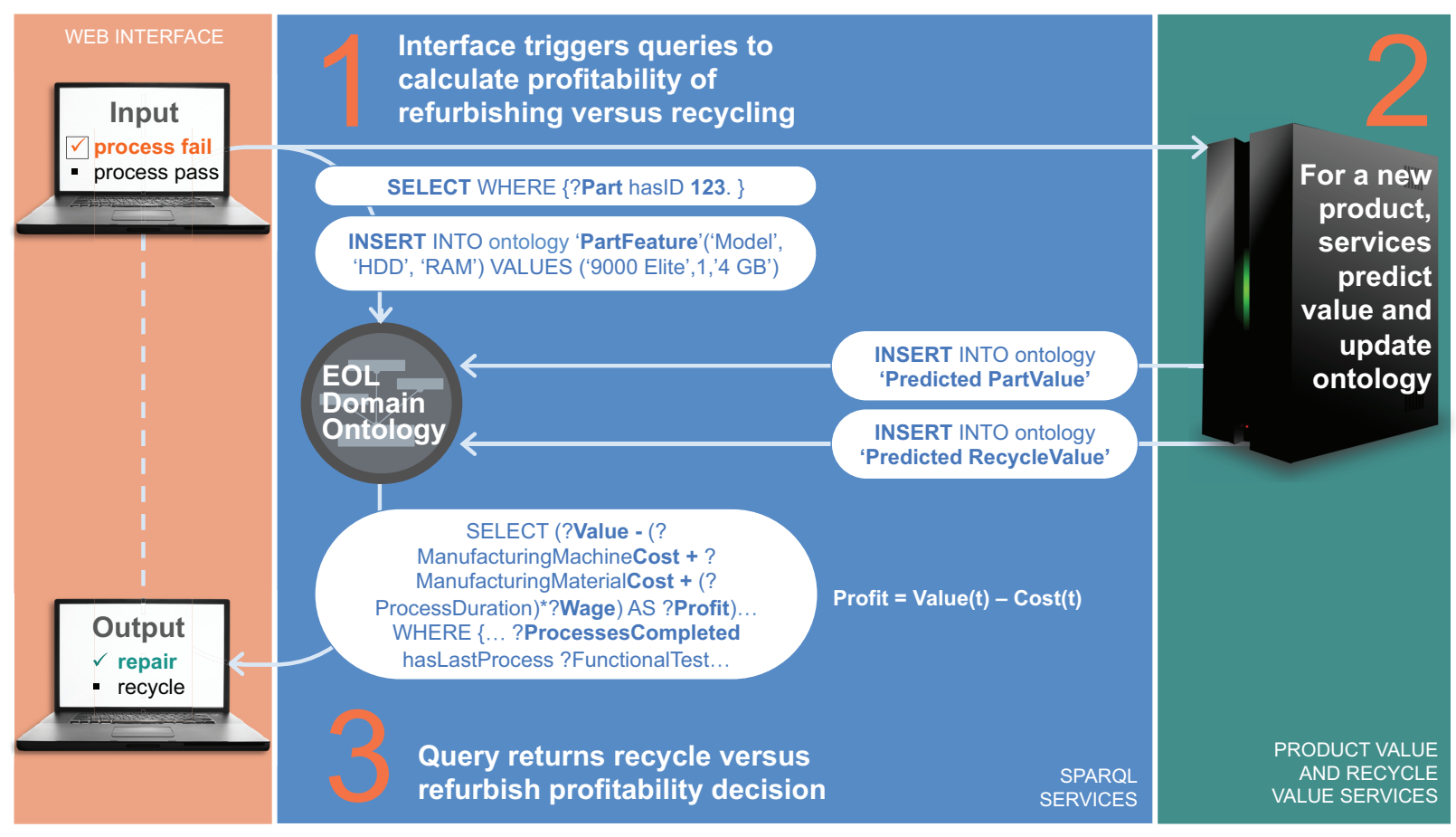

Figure 4: Decision Support Functionality (New Products)

Knowledge gathering for automated population of the sub-domain ontology is performed by the following services:

Value consists of a machine learning and web-scraping service. The machine learning algorithm will take product price training sets that can be provided by the EoL organisation and uses these to predict an appropriate price for similar products. The webscraping service will automate harvesting price information from a range of sources across the web, sanitising the output. A predicted product price is provided to the ontology, with an evaluation of confidence in that price, holding high prestige for data has come from a broker or a trusted sales website and low prestige for a less trusted resource, such as eBay prices for products with limited batch availability.

CheckProductStatus functionality will vary with the sub-domain. For example in the WEEE domain sites indicate a product has been stolen or has specific restrictions such as the software supported or that a phone is locked to a network or the initial factory build.

London Metal Exchange API service will provide ac- cess to the current market recycling prices, according to the specific volume of metals in the product.

SPARQL queries infer processing costs based on component, labour and machine costs. Further details of the SPARQL functionality used to predict profitability are given in section 4.1 .

\subsection{Automated Intelligent Decision Support}

The toolset is designed to support key decisions at the product EoL: should a product be repaired, refurbished or recycled and should a component product be used in the remanufacture, refurbishment or repair of a product assembly or should it be resold or recycled. These decisions depend on equipment, labour and component costs and the market and recycle value of the products. Figure 4 describes the three-step functionality of the proposed toolset in predicting the profitability of a product:

1. The toolset is interrogated for a decision when the user inputs that a new product has been logged or a product is about to go through a higher cost process such as repair. This generates a semantic query to interrogate the ontology for further information on the product. When the user inputs the most recently completed process to the interface, 
this generates an update to the ontology, to calculate processing cost.

2. The interface then triggers all services connected to the service bus to update the ontology, responses are sanitised by the service and a SPARUL request generated to update the triplestore:

(a) The Machine Learning service will request an appropriate price for the product, predicted from a training set of historical broker prices offered for similar products. The machine learning algorithm used by the service is retrained on a monthly basis when a contemporary set of broker prices are submitted to the EoL recovery organisation. A high level of confidence is associated with this prediction in the ontology. If running this service cannot converge on a prediction, the Web Scraping Service is called.

(b) The Web Scraping service searches key websites for current prices of a product, a low level of confidence is associated with this prediction in the ontology.

(c) The Recycling service interrogates the London Metal Exchange API for the price of the metal content of the products. These values are updated in the ontology for use in the calculation of product recycle price.

3. The SPARQL/SPARUL service calculates and outputs to the user interface the predicted remanufacturing and recycle profits using the appropriate pricing of the product from the ontology and the calculation of the cost of any processing and repair completed for the product. The accuracy of this calculation depends on the accuracy and timely update of the predicted appropriate pricing for a product instance via semantic service updates to the ontology.

\subsection{EoL Domain Ontology}

The ontology that is central to the decision support toolset establishes a common and formalised understanding of EoL domain knowledge, applied to the WEEE sub-domain. It is ontologies such as OWL-S that describe service implementation and data access in the semantic web. Cardinality restrictions (Minimum, Maximum and Exactly) were used to define the sets and relationships between individuals for each ontological property.
The ontology was logically validated using the HermiT and Pellet OWL reasoners. The ontology uses RDF and OWL 2 for knowledge engineering and has been designed from the top-down, using BFO 2.0. The BFO classes and properties are indicated with the prefix bfo:, e.g., the class bfo:Thing is the predefined top level class.

BFO stipulates that reality is subdivided for the domain into two ontologies of continuants and occurrents. The continuants ontology represents entities that continue to exist, including substances and parts that also have entitles dependent on their existence such as qualities, functions and roles. The second ontology is one of occurrents, that happen over a period of time, including processes, parts of processes, events and regions of time.

Figure 5 demonstrates the classes of the EoL domain ontology as they inhere from the BFO classes. Subclasses and individuals are added under these top-level classes for a specific EoL sub-domain: WEEE recovery in this paper. The key resources in a recovery facility are classed as object:FacilityResource and consist of Agent, Product, Machine and LabourCost (see Figure 6). In line with the upper-level ontology BFO, processes inhere from occurrents, these are DecisionSupportProcess (that are performed by the DecisionSupport roles, such as Finance or Marketing), PerceptualManualDextrousProcess (performed by ManualDextrous roles, such as Technician or Labourer), StrategicDecisionMakingProcess (performed by DecisionMaker roles, such as Engineer or Supervisor) and process start and end times.

\subsubsection{Domain Continuants: Objects and Dependents}

The Machine class in Figure 5 includes any manufacturing equipment used to process a product and inheres for the validating WEEE subdomain as a PATTestingRig (used for PAT tests) and BlanccoRig (used for data erasure of hard drives) and with the data property assertion hasRunningCost, which is the cost in Pounds Sterling (GBP) per use or per hour.

The Product class has the subclasses Assembly and Component, of which Assembly hasPart some (Component or Assembly). Component is a part of the whole product that is an Assembly of components. For the WEEE subdomain, Component can be a Motherboard, Peripheral, PowerSupply or Storage component, such as a hard disk drive (HDD). Multiple components combine to form an Assembly and multiple assemblies can be combined into the product, which is an Assembly in itself. This is expressed formally in the EoL ontology 

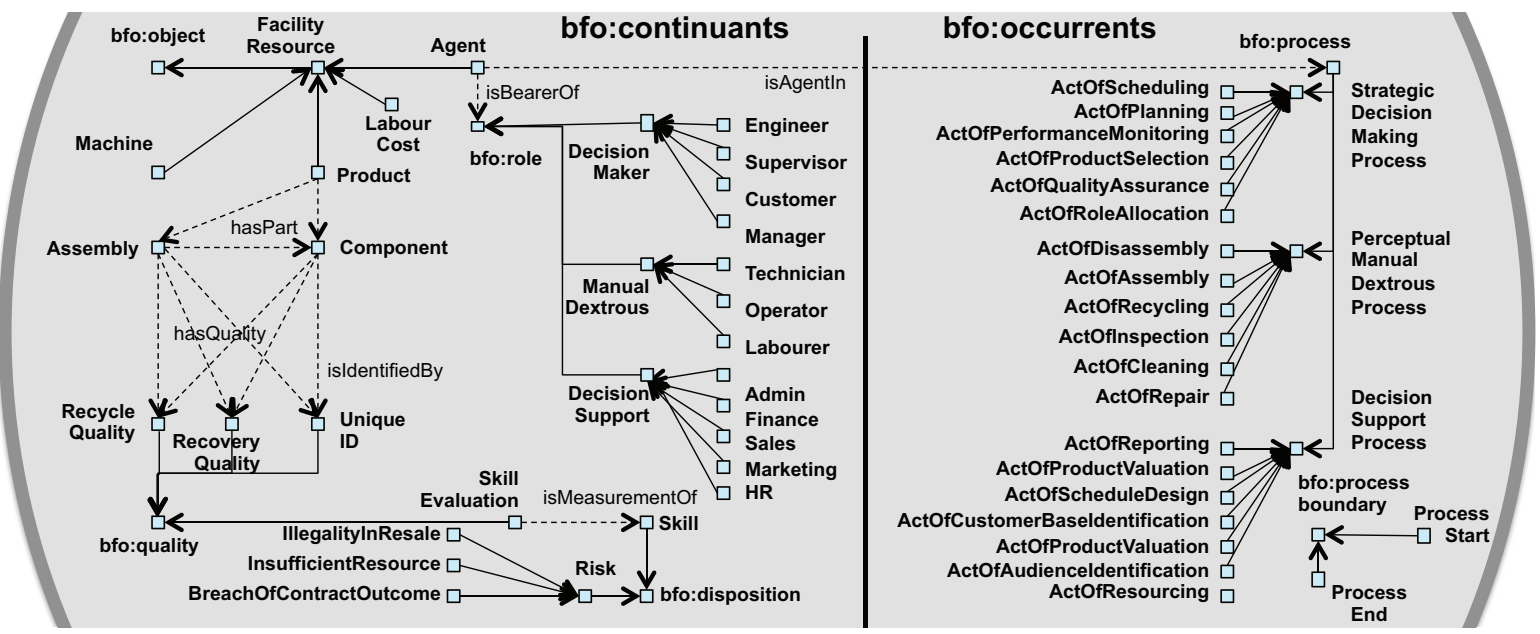

Figure 5: End of Life Domain Ontology

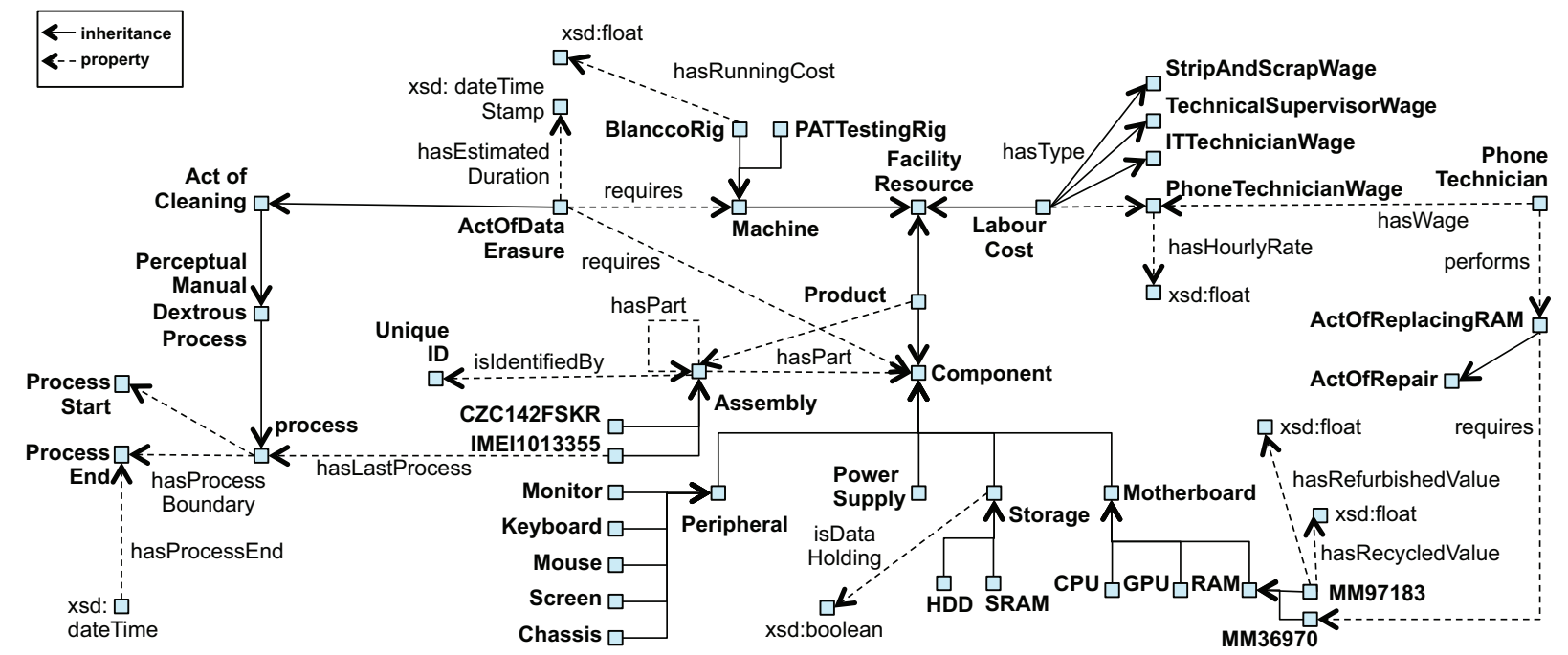

Figure 6: WEEE EoL Sub-Domain Ontology Continuants: Objects 


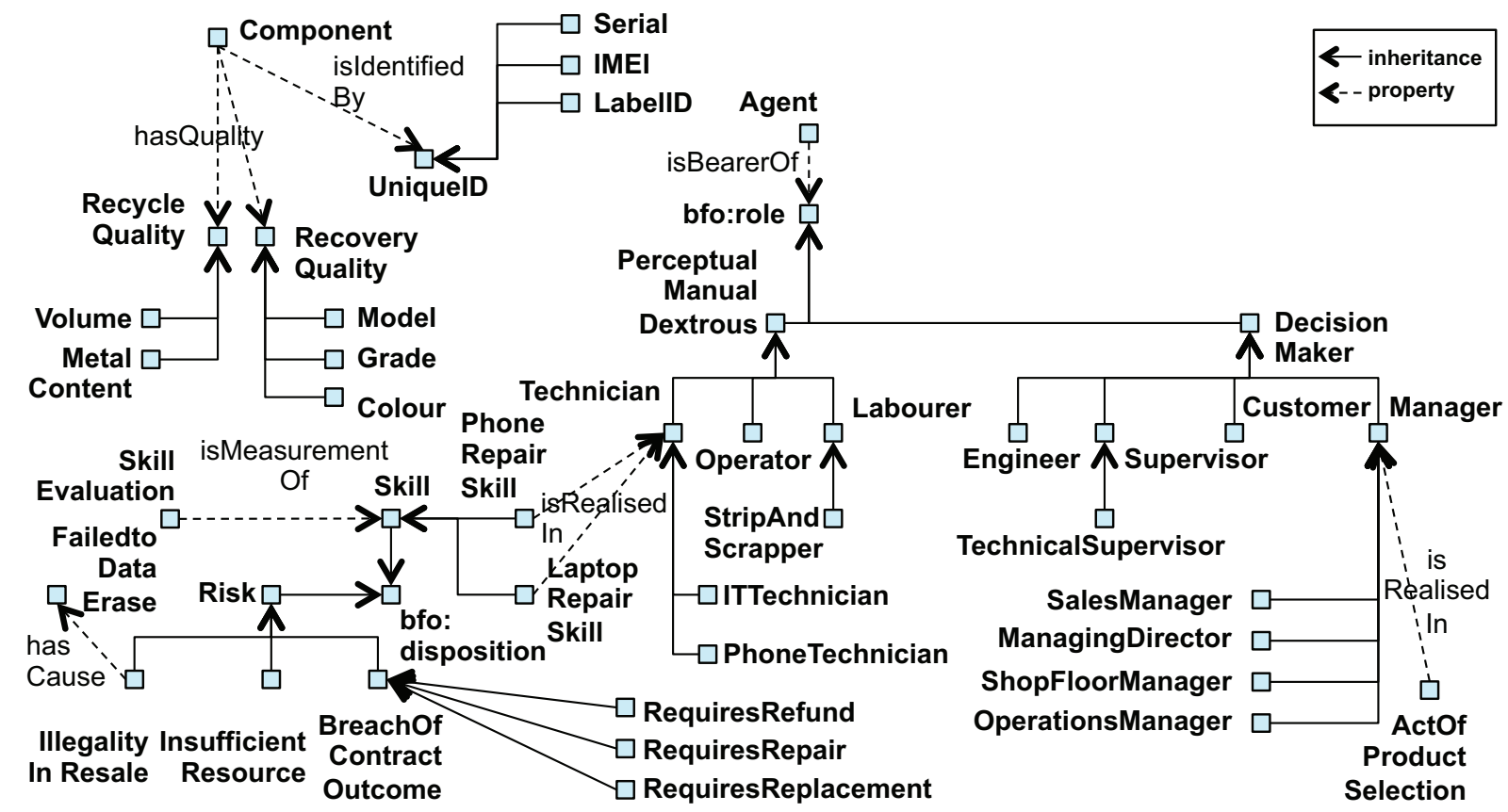

Figure 7: WEEE EoL Sub-Domain Ontology Continuants: Dependents

with the following axiom:

\section{Assembly isSubClassOf \\ hasPart only (Assembly or Component)}

Product also has a bfo:quality that inheres as a RecoveryQuality (Colour, Grade or Model, that influence the appropriate price of the refurbished product) or RecycleQuality (MetalContent or Volume, that influence the price of the recycled product). As shown in Figures 6- 7, each Component and Assembly has a UniqueID number that has a type: IMEI, for a phone or LabelID or Serial number and is used to instantiate individuals:

\section{Product isSubClassOf}

\section{isIdentifiedBy exactly 1 UniquelD}

As shown in Figures 5- 6, the Agent is the bearerOf a role, such as the ManualDextrous role of Technician. The role is instantiated for the sub-domain, for example a PhoneTechnician for WEEE recovery, who performs a process such as ActofRepair:ActofReplacingRAM. Each Role has an associated wage, for example the PhoneTechnician has PhoneTechnicianWage that is an instantiation of the class LabourCost and does not include factory overheads. The datatype property hasHourlyRate is then used to assign a value in GBP. A process may require the use of some Machine or some
Component, for example ActOfDataErasure requires the Machine:BlanccoRig and ActOfRepacingRAM requires the component RAM:MM36970.

The roles and role-driven processes are defined as DecisionMaker, ManualDextrous and DecisionSupport, using occupational classifications and definitions of the UK Office for National Statistics [36], the United Nations International Labour Office [37] and the authors in [38]. DecisionMaker, can be an external decision maker, such as a Customer whose decision drives demand, and internal decision makers, such as a Managers who responds to or makes predictions on demand. DecisionMaker:Manager is an internal higher level decision makers who strategise and control budgets to enable resource allocation, set and monitor performance targets and identify opportunities for improvement and expansion. DecisionMaker:Supervisor enacts plans set by managers according to economy, staff, and environment, overseeing day to day operations and training. DecisionMaker:Engineer contributes to and makes design decisions, relating to new and improvements to existing designs of facility and equipment based on targets set by managers.

The next type of role defined is ManualDextrous, requiring manual manipulation, or high levels of visual perception. The ManualDextrous:Operator runs and monitors multi-function machinery and equipment, util- 
ising a range of resources such as computerised controls, monitors, and physical artefacts. There may be some functional overlap between Operator and Technician who operates, controls and monitors machines and computerised controls but will also optimise processes and detect malfunctions, read gauges, meters and charts and troubleshoot and take corrective action. The ManualDextrous:Labourer provides support to operators and technicians through material handling tasks which can be physically demanding including picking, packing, sorting, loading and unloading from vehicles and conveying goods to work areas using human powered vehicles. Labourers may clean machinery, tools and equipment.

The third type of role is DecisionSupport that often includes repetitive tasks such as data entry, paying bills or collecting customer information and simple decisions that could be supported by automation. The roles in this category are Administration that may include preparation of and reporting on production schedules, Finance that may include forecasting production capacity and performance, HumanResources that may make decisions on managing and reporting on flows of work and performance, Marketing that involves some customer relationship management with a focus on promoting customer interest and satisfaction and Sales that includes some direct interaction with the customer to facilitate purchasing.

Skill is a bfo:disposition that inheres for the validating subdomain, for example as LaptopRepairSkil or PhoneRepairSkill and that isRealisedIn a particular role, in these examples a Technician. The quality:SkillEvaluation is then a measurement of the Skill that can have the level Awareness, EntryLevel, Experienced or ExpertKnowledge.

Products also have a bfo:disposition (see Figure 7) in the form of a Risk, the realisation of the risk will have a consequence for profit recovery from that product. The subclasses of realised risk are IllegalityInResale, InsufficientResource and BreachOfContractOutcome. The types of BreachOfContractOutcome in WEEE EoL recovery are either RequiresRefund, RequiresRepair or RequiresReplacement. There may be illegality in the resale of goods where the WEEE recovery organisation has FailedtoDataErase ${ }^{4}$ or ResoldStolenGoods. There is a risk of InsufficientResource, for example if the SkillEvaluation demonstrates insufficient skill for the repair or a required machine, tool or component are not present.

${ }^{4}$ Under the UK Data Protection Act 2018

\subsubsection{Processes: Domain Occurrents}

As discussed in Section 4.2.1, the role-driven processes are defined using occupational classifications [36, 37, 38]. The occurrents ontology consists of the processes applied in EoL recovery and process boundaries, that are instantiated to record an xsd:datetimestamp for ProcessStart and ProcessEnd. These are used to identify the mean time to complete each process for the WEEE manufacturing sub-domain as well as the time a process was started and completed for a particular product instance. The mean value can be used to infer the expected time to complete a future process and the recorded values in calculating the LabourCost of all processing conducted on a product so far. The occurrents ontology is also used to define the ordering of processes using the isFollowedBy property, as shown in Figure 8.

Processes are defined as DecisionSupportProcess, StrategicDecisionMakingProcess or PerceptualManualDextrousProcess and relate to the roles that perform them as seen in Figure 5. ActofAudienceIdentification is realisedIn the role Marketing. The ActofCustomerBaseIdentification is realisedIn the role Sales. ActofProductValuation, ActofReporting and ActofScheduleDesign will be perfomed by the Finance or Administration roles. These processes are to be performed by the system proposed in this paper, in particular ActofProductValuation, ActofCustomerBaseIdentification and some ActofReporting.

ActofPerformanceMonitoring staff, ActofPlanning work flows, ActofProductSelection for purchase, ActofQualityAssurance of resale products, ActofResourcing of components and materials, ActofRoleAllocation to new staff and ActofScheduling processes are the generic processes performed in strategic decision making in the EoL domain. For example the ActOfProductSelection isRealisedIn the Manager role.

Figure 2 demonstrates that ActofInspection is one of the first processes performed when a product arrives at a facility and is also performed prior to any high cost process such as ActofRepair. ActOfAssetTracking, ActOfFunctionalTesting and ActOfVisualInspection are the types of inspection that are performed in the WEEE EoL recovery sub-domain. As a product passes through these processes the instance is updated with the data property assertion of previousProcess.

ActofAssembly and ActofDisassembly are performed by an Agent who isBearerOf the role:ManualDextrous. This is instantiated for the sub-domain, for example in WEEE EoL the PhoneTechnician will perform ActofPhoneDisassembly. ActofRe- 


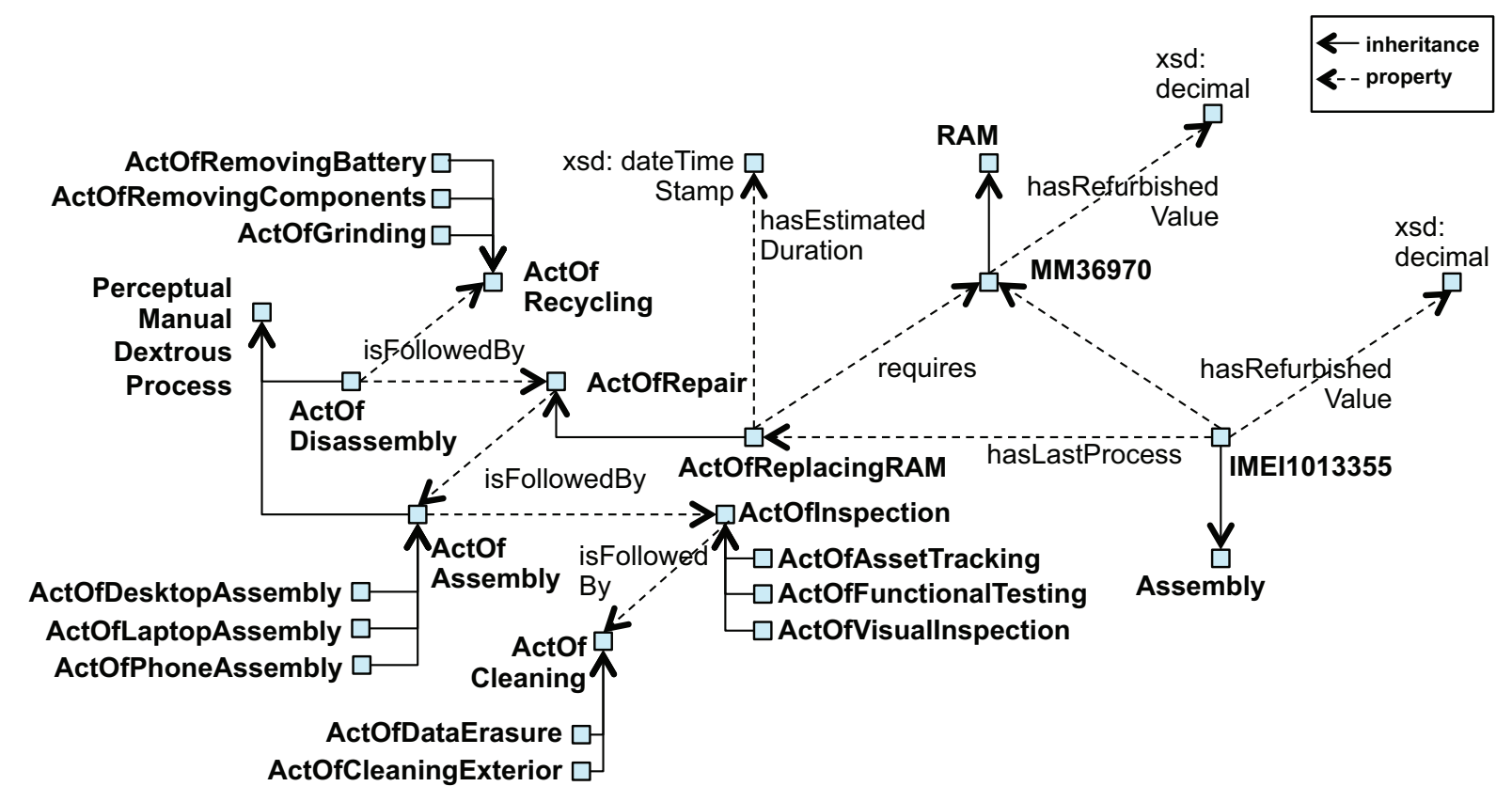

Figure 8: WEEE EoL Sub-Domain Ontology Occurents

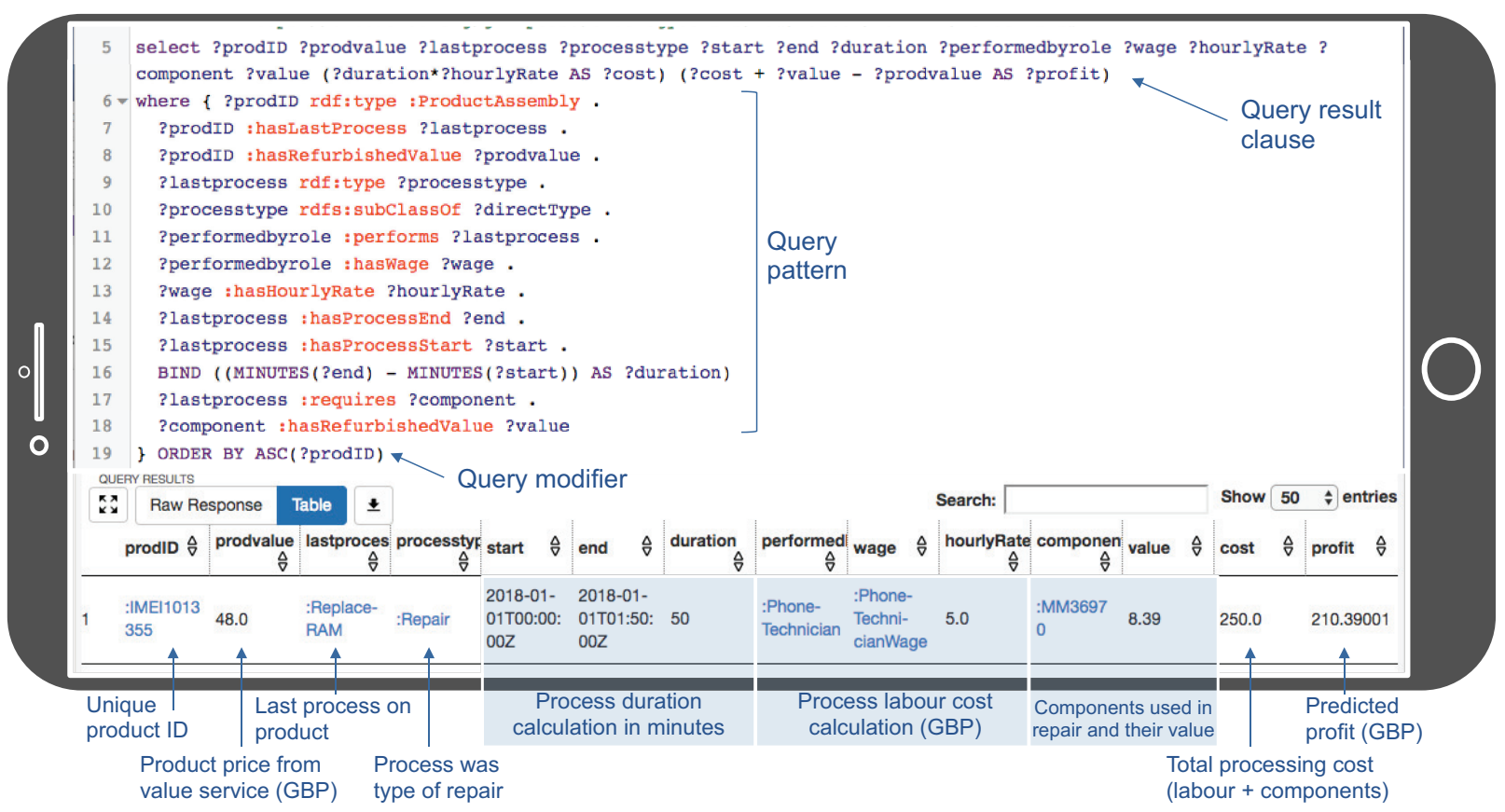

Figure 9: Validation: SPARQL Query Returning Profitability of a Product

pair may include replacing damaged components, ActofCleaning includes ActOfCleaningExterior and ActOfDataErasure of device storage. ActofRecycling of WEEE is performed by a Labourer:StripandScrapper (see Figure 7- 8). In the WEEE EoL domain, the order of most of the processes is strictly defined. The processes that occur will depend on the state and quality of the product on inspection. For instance, the 


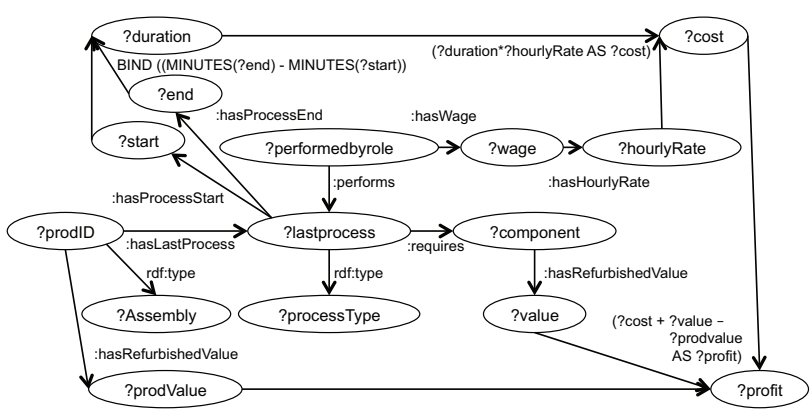

Figure 10: Validation: Query Graph

disassembly process can only be followed by recycling or repair:

\section{Disassembly isSubClassOf}

isFollowedBy only (ActOfRecycling

or ActOfRepair)

\section{Toolset Validation}

The toolset was validated for remote deployment and querying of the linked-data and initial demonstration of the decision-making capabilities. An RDF representation of the linked semantic dataset has been remotely hosted from a public SPARQL 1.1 [39] endpoint, created using Apache JenaTDB (JenaTDB). An endpoint is a client/server architecture that can accept queries and return results via the HTTP protocol. The EoL decision support toolset has initially been validated by querying this remotely hosted ontology. An example query and response is shown in Figure 9 along with the traversal of the query in Figure 10.

Remote querying was used to determine the profitability of a particular part when the unique identifier of the part was input into the system. The query result clause and query pattern specify the order and parameters that the query will return from the dataset. In order to predict the cost of recovering a product the following were taken into account: the processing conducted, wage of the worker involved in processing, price of any components involved in the process and running cost of any machine involved in the process.

The names of the variables in the SPARQL query are not the same as the URI's but relevant shortened names are used due to the complexity of the query, for example ProcessEnd stands in for ?end . The query graph (Figure 10) demonstrates the traversal of the subjects, predicates and objects that match the query pattern. For a provided unique identifier of the part IMEI1013355 (?prodid) the query requests that where a:performs lastprocess on the ProductAssembly and a:hasWage and also a:requires component which hasRefurbishedValue the values specified in the select statement, or query result clause are returned. MINUTES takes an xsd:dateTime and returns the minutes part of the argument, converting it into an xsd:integer. BIND allows the value of the difference between start and end time to be assigned to a variable, in this case duration. Three of the returned variables: duration, cost (Processing duration * Wage of agent) and prof it (Total processing cost + Component value - Product price) have also been aggregated from the data.

The appropriate product price (prodvalue) is that provided by the Value Service. The toolset infers a final profit can still be made on this product of GBP £210.39. At this point the operator would be advised by the web front end to continue processing this product and what the next process should be - in this case, a repair or assembly process.

\section{Conclusions and Future Work}

This paper has presented the design of an EoL semantic decision support toolset and the initial implementation and evaluation in the form of an EoL domain ontology instantiated and validated for the WEEE sub-domain. Other existing decision support systems in this domain have failed to consider harnessing knowledge from a range of online and offline sources, that can ensure accurate predictions of appropriate product price are continuously updated. This toolset has been designed to use a formal representation of the knowledge, supporting interoperability, reusability and scalability to being used across various EoL processing subdomains. The semantic service architecture design and the developed EoL Domain Ontology enable interoperable support and sustainability of a novel suite of realtime profitability and recyclability business decisions services. These decisions are complex due to EoL product and market diversity. The proposed toolset supports these time-critical decisions, allowing knowledge to be repeatedly re-evaluated, to better support high-cost processing such as product refurbishment.

Future work will extend the toolset to include user and developer interfaces and semantic machine learning and web scraping services. These services will function in a priority queue to extract information from historical datasets and external websites, ordered by level of trust in the pricing data using an attack resistant trust metric 
to quantify prediction confidence. The next service in the queue will be called when the previous cannot predict a appropriate product price. The addition of a probabilistic Bayes Network will provide information on the accuracy of the predicted output. The domain ontology will be further extended to support decisions enabling work to be efficiently directed to agents with appropriate skill bases and levels of experience. The ontology will also be validated in the automotive and wind turbine EoL sub-domains.

\section{Acknowledgment}

The authors would like to thank the EPSRC for the funding of the project Adaptive Informatics for Intelligent Manufacturing (EP/K014137/1).

[1] K. Xia, L. Gao, L. Wang, W. Li, K.-M. Chao, A Semantic Information Services Framework for Sustainable WEEE Management Toward Cloud-Based Remanufacturing, Journal of Manufacturing Science and Engineering 137 (6) (2015) 061011

[2] D. Parker, K. Riley, S. Robinson, H. Symington, J. Tewson, K. Jansson, S. Ramkumar, D. Peck, Remanufacturing Market Study, European Remanufacturing Network (2015) 145.

[3] A. F. Chalmers, Chapter 6: Sophisticated falsificationism, novel predictions and the growth of science, What is this thing called science? 5 (4) (1999) 74-81.

[4] T. B. Sheridan, R. Parasuraman, Human-Automation Interaction, Reviews of Human Factors and Ergonomics 1 (1) (2005) 89-129.

[5] B. Schmidt, L. Wang, D. Galar, Semantic framework for predictive maintenance in a cloud environment, Procedia CIRP $62(\mathrm{Cm})(2017)$ 583-588.

[6] S. H. Hong, S. K. Lee, J. H. Yu, Automated management of green building material information using web crawling and ontology, Automation in Construction 102 (January) (2019) 230244.

[7] C. J. Pearson, C. B. Mayhorn, Who should i trust (human vs. automation)? the effects of pedigree in a dual advisor context, in: Proceedings of the 20th Congress of the International Ergonomics Association (IEA 2018), Springer International Publishing, 2019, pp. 10-17.

[8] K. A. Hoff, M. Bashir, Trust in automation: Integrating empirical evidence on factors that influence trust, Human Factors 57 (3) (2015) 407-434.

[9] F. Burstein, S. A. Carlsson, Decision Support Through Knowledge Management, Springer Berlin Heidelberg, Berlin, Heidelberg, 2008, pp. 103-120.

[10] C. Palmer, Z. Usman, O. C. Junior, A. Malucelli, R. I. M. Young, Interoperable manufacturing knowledge systems, International Journal of Production Research 0 (0) (2017) 1-20.

[11] P. Delir Haghighi, F. Burstein, A. Zaslavsky, P. Arbon, Development and evaluation of ontology for intelligent decision support in medical emergency management for mass gatherings, Decision Support Systems 54 (2) (2013) 1192-1204.

[12] A. Matsokis, D. Kiritsis, Computers in Industry An ontologybased approach for Product Lifecycle Management, Computers in Industry 61 (8) (2010) 787-797.

[13] K. Baclawski, A. Ghoneimy, E. S. Chan, K. Gross, Framework for Ontology-Driven Decision Making (2017) 1-35.

[14] M. Alkahtani, A. Choudhary, A. De, J. Harding, A Decision Support System based on Ontology and Data mining to Improve
Design using Warranty Data, Computers \& Industrial Engineering.

[15] S. Hou, H. Li, Y. Rezgui, Ontology-based approach for structural design considering low embodied energy and carbon, Energy and Buildings 102 (2015) 75-90.

[16] H. B. Jun, D. H. Lee, J. G. Kim, D. Kiritsis, Heuristic algorithms for minimising total recovery cost of end-of-life products under quality constraints, International Journal of Production Research 50 (19) (2012) 5330-5347.

[17] S. J. Cho, H. B. Jun, D. Kiritsis, Heuristic algorithms for maximising the total profit of end-of-life computer remanufacturing, International Journal of Production Research 55 (5) (2017) 1350-1367.

[18] S. Behdad, A. S. Williams, D. Thurston, End-of-Life Decision Making With Uncertain Product Return Quantity, Journal of Mechanical Design 134 (10) (2012) 100902.

[19] P. S. P. Shrivastava, H. Zhang, J. L. J. Li, a. Whitely, Evaluating obsolete electronic products for disassembly, material recovery and environmental impact through a decision support system, Proceedings of the 2005 IEEE International Symposium on Electronics and the Environment, 2005. (2005) 221-225.

[20] V. Mascardi, V. Cordì, P. Rosso, P. R. Viviana Mascardi, Valentina Cordì, A Comparison of Upper Ontologies, in: WOA 2007, th AIIA/TABOO Joint Workshop "From Objects to Agents": Agents and Industry: Technological Applications of Software Agents, 2007, pp. 55-64.

[21] B. Smith, M. Ashburner, C. Rosse, J. Bard, W. Bug, W. Ceusters, L. J. Goldberg, K. Eilbeck, A. Ireland, C. J. Mungall, T. O. B. I. Consortium, N. Leontis, P. Rocca-Serra, A. Ruttenberg, S.-A. Sansone, R. H. Scheuermann, N. Shah, P. L. Whetzel, S. Lewis, The OBO Foundry: coordinated evolution of ontologies to support biomedical data integration, Nature Biotechnology 25 (2007) 1251.

[22] L. Temal, A. Rosier, O. Dameron, A. Burgun, Mapping BFO and DOLCE (Icd) (2010) 1065-1069.

[23] S. K. Semy, M. K. Pulvermacher, L. J. Obrst, Towards the Use of an Upper Ontology for U . S . Government and Military domains : An Evaluation, Tech. Rep. September (2004).

[24] G. Adamson, L. Wang, M. Holm, P. Moore, Cloud manufacturing-a critical review of recent development and future trends, International Journal of Computer Integrated Manufacturing 30 (4-5) (2017) 347-380.

[25] L. Xu, W. He, S. Li, Internet of Things in Industries: A Survey, IEEE Transactions on Industrial Informatics PP (4) (2014) 1-11.

[26] T. Vitvar, M. Moran, M. Zaremba, T. Vitvar, M. Moran, M. Zaremba, Services Haller, Paavo Kotinurmi " Semantic SOA to Promote Semantic SOA to Promote Integration of Heterogeneous B2B Services (2007) 2-6.

[27] D. Karastoyanova, B. Wetzstein, T. Van Lessen, D. Wutke, J. Nitzsche, F. Leymann, Semantic Service Bus : Architecture and Implementation of a Next Generation Middleware (2007) 347-354.

[28] D. Hutchison, J. C. Mitchell, J. Famaey, S. Latré, J. Strassner, F. De Turck, An ontology-driven semantic bus for autonomic communication elements, in: Lecture Notes in Computer Science (including subseries Lecture Notes in Artificial Intelligence and Lecture Notes in Bioinformatics), Vol. 6473 LNCS, 2010, pp. 37-50.

[29] P. Holtewert, R. Wutzke, J. Seidelmann, T. Bauernhansl, Virtual fort knox federative, secure and cloud-based platform for manufacturing, in: Procedia CIRP, Vol. 7, 2013, pp. 527-532.

[30] M. Bone, M. Blackburn, T. Hagedorn, J. Dzielski, B. Kruse, I. Grosse, Toward an Interoperability and Integration Framework to Enable Digital Thread, Systems 6 (4) (2018) 46.

[31] H. Walzel, M. Vathoopan, A. Zoitl, A. Knoll, An Approach for 
an Automated Adaption of KPI Ontologies by Reusing Systems Engineering Data, IEEE International Conference on Emerging Technologies and Factory Automation, ETFA 2019-September (2019) 1693-1696.

[32] S. Hachem, T. Teixeira, V. Issarny, Ontologies for the Internet of Things, ACMIFIPUSENIX 12th International Middleware Conference (2011) 3:1-3:6.

[33] F. Roda, E. Musulin, An ontology-based framework to support intelligent data analysis of sensor measurements, Expert Systems with Applications 41 (17) (2014) 7914-7926.

[34] F. X. Xu, X. H. Liu, W. Chen, C. Zhou, B. W. Cao, An ontology and AHP based quality evaluation approach for reuse parts of end-of-life construction machinery, Mathematical Problems in Engineering 2018.

[35] S. Chen, J. Yi, H. Jiang, X. Zhu, Ontology and CBR based automated decision-making method for the disassembly of mechanical products, Advanced Engineering Informatics 30 (3) (2016) 564-584.

[36] Office for National Statistics (ONS), SOC2010 volume 1: structure and descriptions of unit groups - Office for National Statistics (2010).

[37] International Labour Office, International Standard Classification of Occupations (ISCO-08): Structure, group definitions and correspondence tables, Volume 1, Tech. rep., Geneva (2012).

[38] C. B. Frey, M. Osborne, The Future of Employment (2013).

[39] S. Harris, A. Seaborne, E. Prud'hommeaux, Sparql 1.1 query language, W3C recommendation 21 (10) (2013) 778 